\section{Cureus}

Received 03/28/2019

Review began 03/31/2019

Review ended 04/03/2019

Published 04/12/2019

\section{(c) Copyright 2019}

Lofgren et al. This is an open access article distributed under the terms of the Creative Commons Attribution License CC-BY 3.0., which permits unrestricted use, distribution, and reproduction in any medium, provided the original author and source are credited.

\title{
Abdominal Wall Abscess Secondary to Cholecystocutaneous Fistula via Percutaneous Cholecystostomy Tract
}

Daniel H. Lofgren ${ }^{1}$, Sugam Vasani ${ }^{2}$, Victorico Singzon ${ }^{3}$

1. Otolaryngology, McLaren Oakland Hospital, Pontiac, USA 2. General Surgery, United Hospital Center, Bridgeport, USA 3. Family Medicine, United Hosptial Center, Bridgeport, USA

Corresponding author: Daniel H. Lofgren, daniel.lofgren@mclaren.org

\section{Abstract}

Cholecystocutaneous fistulas (CCFs) are an increasingly rare consequence of chronic gallbladder inflammation and disease. Historically, they were commonly noted in the literature by Courvoisier, Naunyn, and Bonnet in the late 1800s. Due to improvements in diagnostic imaging and treatment options in the last century, there has been a marked decrease in the incidence of the CCF cases in the literature. From the late 1890s to 1949, there were only 37 cases presented in the literature; only 28 cases have been reported since 2007. This case is only the second noted CCF in the literature that followed percutaneous cholecystostomy drain placement and removal.

General surgery was consulted on a 60-year-old morbidly obese female, who presented to the emergency department after one week of fever, right upper quadrant (RUQ) pain, nausea, emesis, and shortness of breath. She had a history of acute cholecystitis treated with a cholecystostomy tube the year prior, but after the removal of the tube, she was lost to follow up. She was found to have a $14 \mathrm{~cm}$ x $5 \mathrm{~cm}$ fluctuant abdominal wall abscess in her RUQ that was treated with incision and drainage (I\&D) along with ertapenem. She continued to improve until day 7 post-I\&D when yellowish-green discharge was noted draining from the wound. After a negative hepatobiliary iminodiacetic acid scan, a follow-up abdominal computed tomography (CT) showed a contracted gallbladder with fistula formation underlying the abscess location, near the site of her prior cholecystostomy tube. A robotic-assisted cholecystectomy was performed, which improved the wound drainage, and the patient was discharged home 5 days later.

This case is the only noted CCF presenting as a RUQ abscess after cholecystostomy drain placement. The patient lacks follow up after the removal of her percutaneous drain and continued inflammation in the gallbladder provided perfect nidus for the fistula formation. As seen in other CCF patients, cholecystectomy is the treatment of choice, and this case was successfully treated via robotic-assisted cholecystectomy with adhesiolysis.

Categories: Gastroenterology, General Surgery, Anatomy

Keywords: cholecystocutaneous fistula, percutaneous cholecystostomy, abscess, cholecystectomy, abdominal pain, non-compliance

\section{Introduction}

Biliary fistulas are pathologic connections formed from chronic inflammation between the gallbladder and adjacent organs [1-2]. Historically, biliary fistulas were a common outcome of

How to cite this article

Lofgren D H, Vasani S, Singzon V (April 12, 2019) Abdominal Wall Abscess Secondary to 
longstanding gallbladder inflammation and disease [3-5]. With improving diagnostic technology and treatment, biliary fistulas are being reported to a lesser extent in the literature [6]. External biliary fistulas (EBFs), which generally connect the gallbladder to the skin, subcutaneous tissues, and abdominal wall, are increasingly rare in occurrences. A cholecystocutaneous fistula (CCF) connects the gallbladder to the skin, and is a form of EBF that has been reported with less frequency in the last century due to improving medical diagnostic techniques and identification. A total of 169 cases were reported by Courvoisier in the 19th century [7-8]. From 1900 to 1991, there were only a reported 65 cases of EBF in the literature [4,9-10]. From 1890 to 1949, only 37 cases were found in the medical literature. Since 2007, only 28 cases have been published [5-6]. These fistulas can be secondary to abdominal procedures or other traumatic causes. The cutaneous openings can drain bile and gallstones through external sinuses and have been noted at various locations including the right upper quadrant, umbilicus, and even in the gluteal folds [7,11]. Even more uncommon are the EBFs associated with percutaneous cholecystostomy drains, with only one other case report seen currently in the literature [12]. The case below is the first noted CCF presenting as an abscess following percutaneous cholecystostomy drain removal.

\section{Case Presentation}

A 60-year-old morbidly obese, white female was admitted to the floor with one week of worsening shortness of breath, right upper quadrant (RUQ) pain, nausea, emesis, and a fever. General surgery was consulted after a large, fluctuant and erythematous mass was visualized on the RUQ abdominal wall. The patient noted a history of severe cholecystitis the year prior that was managed by a percutaneous cholecystostomy drain. After the drain was removed, she was lost to follow up. Initial laboratory evaluation revealed: leukocytosis with $91 \%$ neutrophils (white blood cell count 14,800, reference range 3500 - $10300 \mathrm{~mm} 3$ ), international normalized ratio of 2.51 (reference range $0.90-1.10$ ), alkaline phosphatase of 162 (reference range 20-130 $\mathrm{U} / \mathrm{L})$; lactic acid, aspartate aminotransferase, and alanine aminotransferase were within normal limits. A computed tomography (CT) scan of the abdomen revealed a $14 \mathrm{~cm} \mathrm{x} 5 \mathrm{~cm}$ abdominal wall fluid and air collection suspicious for an abscess in the RUQ as seen in Figures 1-2.

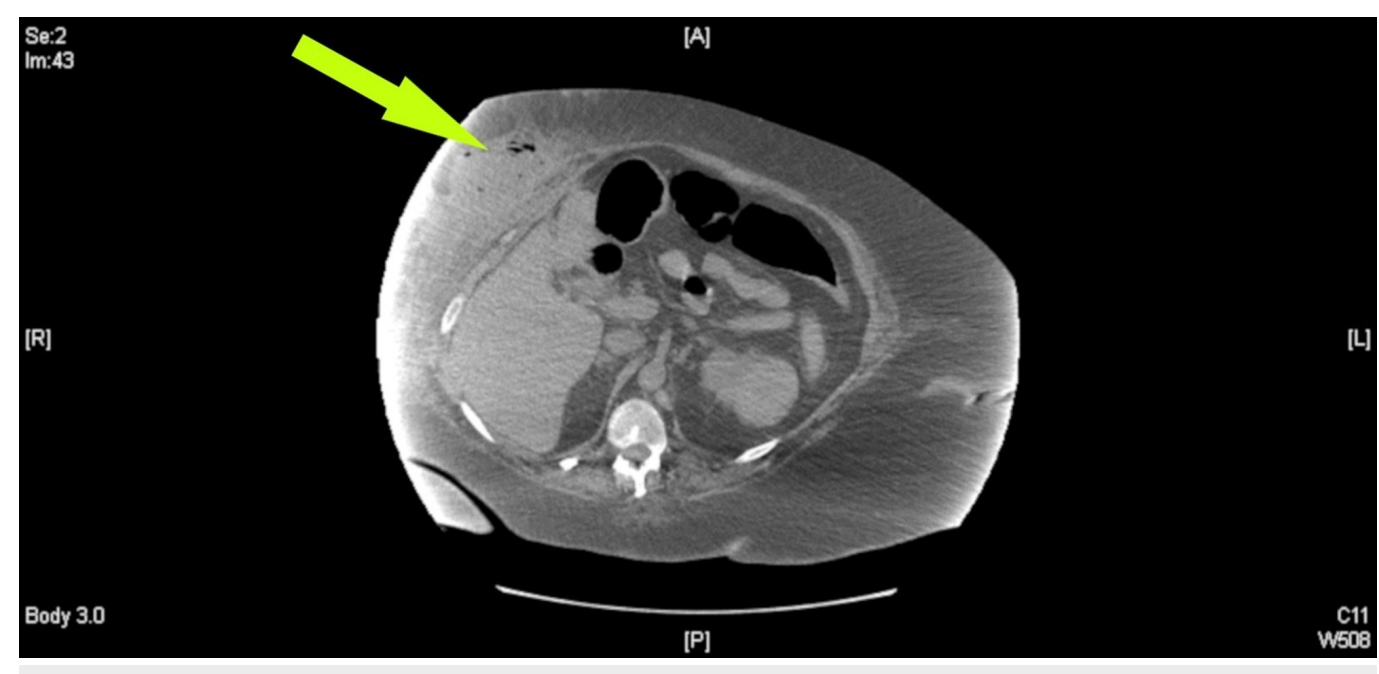

FIGURE 1: Axial view of noncontrast computed tomography of the abdomen. Arrow demonstrates subcutaneous right upper quadrant fluid collection with associated air-fluid levels and local fat stranding. Superiormost image of the abscess site. 


\section{Cureus}

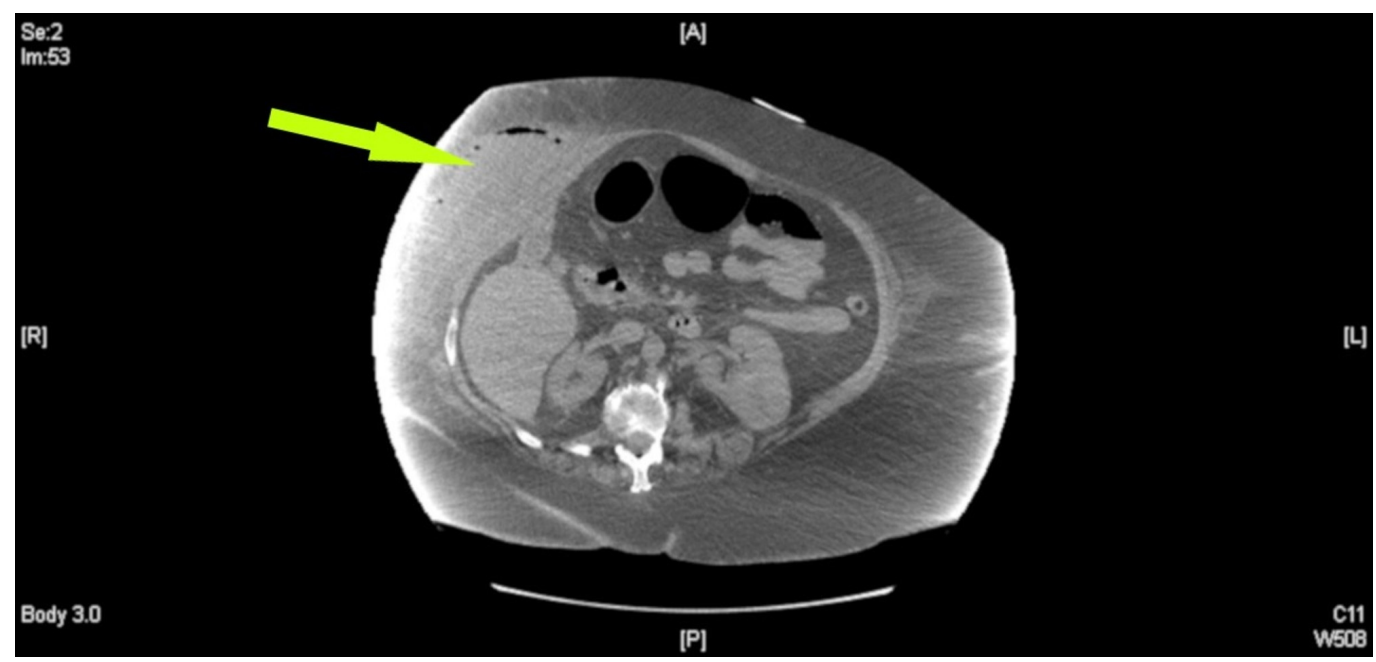

FIGURE 2: Additional axial view of noncontrast computed tomography of abdomen. Arrow shows large subcutaneous

fluid collection noted over right upper quadrant with associated fat stranding and air-fluid levels.

Incision and drainage (I\&D) of the abscess were performed with a cruciate incision over the indurated RUQ of the abdomen as seen in Figure 3. One hundred and fifty milliliters of the purulent material was irrigated via pulsed lavage with normal saline. Intraoperative wound cultures revealed Escherichia coli along with Bacteroides fragilis, which were treated with ertapenem. The patient continued to improve with daily packing changes until day seven post operation, when she was noted to have yellow-green discharge draining from the wound site and increasing tenderness with packing changes. Figure 4 demonstrates the wound and discharge appearance. There was a concern that the fluid was bile rather than an infection due to the location and size of the initial abscess. A hepatobiliary iminodiacetic acid scan was performed but failed to demonstrate a biliary fistula tract. A second CT scan with oral contrast was ordered and after further review, it was noted that her gallbladder was severely contracted and located near the abscess site, which supported the idea of a CCF. This study was compared with a prior CT taken after percutaneous cholecystostomy tube placement in 2016. It was noted that the current abscess site was located near the drain placement site. Due to worsening drainage from the wound and the probable fistula formation, a robotic-assisted cholecystectomy with intraoperative cholangiogram was performed. 


\section{Cureus}

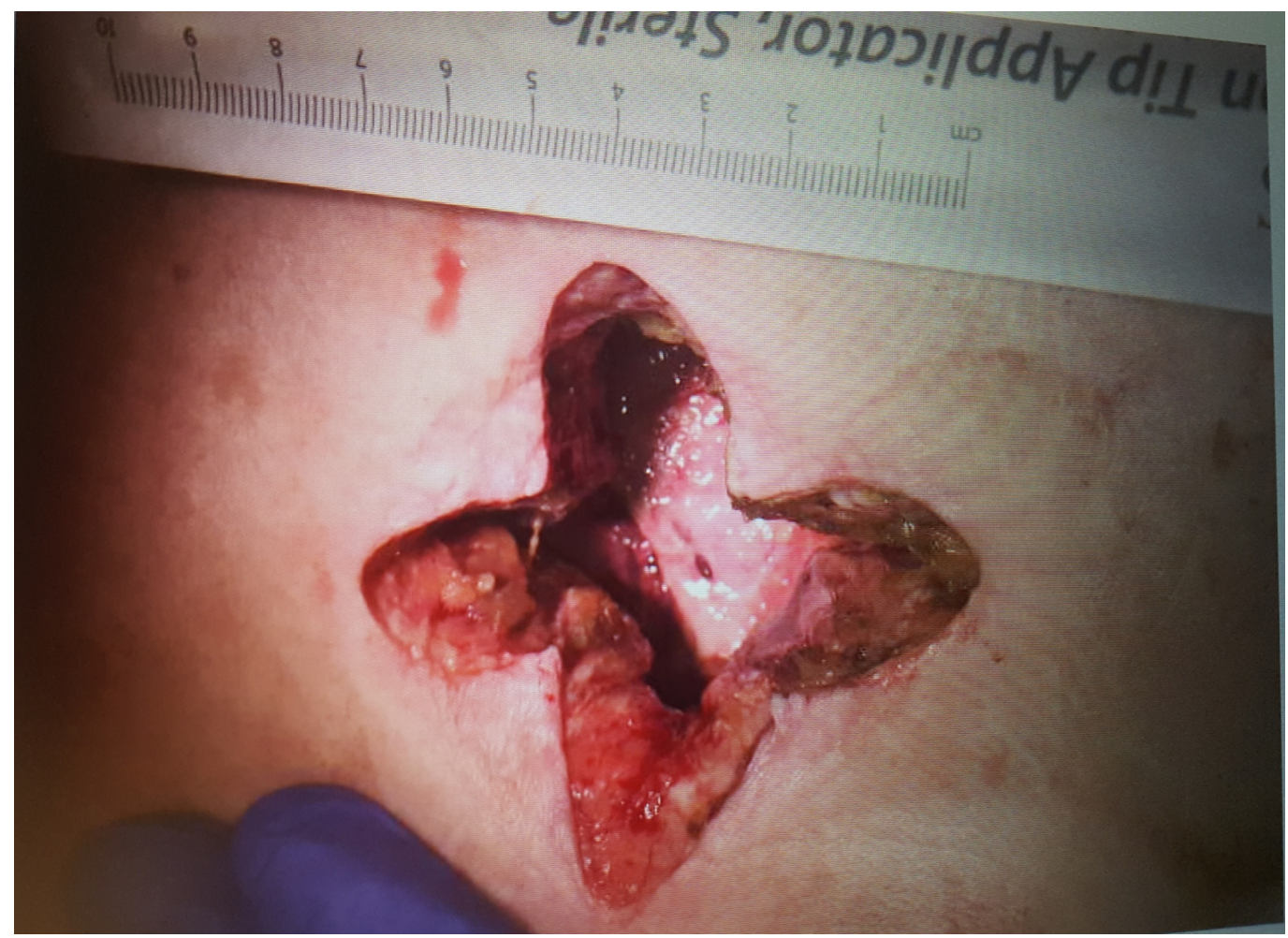

FIGURE 3: Right upper quadrant cruciate incision after pulse lavage with normal saline. 


\section{Cureus}

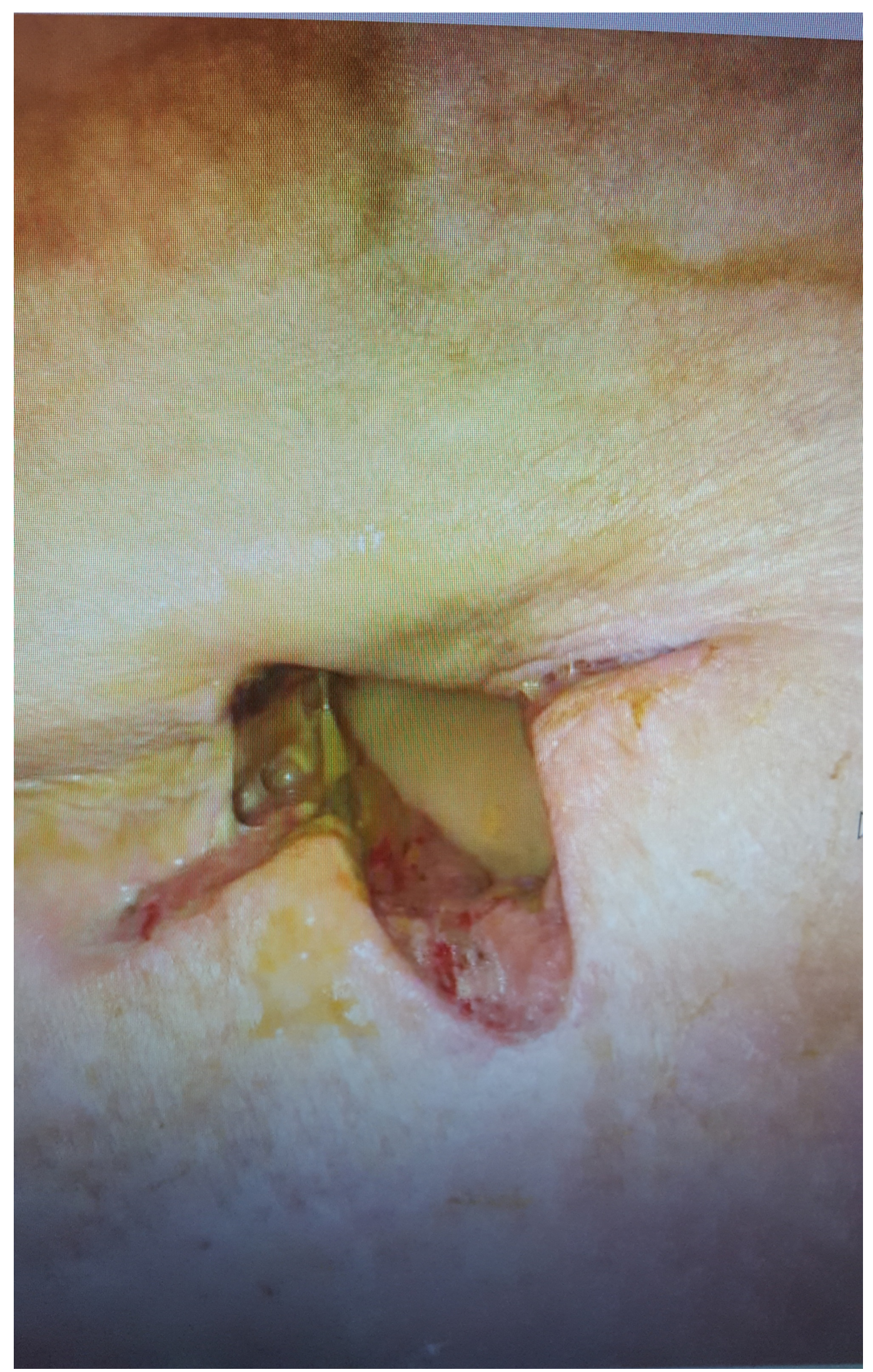

FIGURE 4: Incision site on postoperative day 7. Note the bilious appearing fluid draining from site during packing changes. 


\section{Cureus}

Once inside the abdomen, dense adhesions were visualized in the RUQ of the peritoneal cavity along with a laterally adhered, contracted gallbladder. Two hours of adhesiolysis was performed due to the numerous dense adhesions in the RUQ. An intraoperative cholangiogram confirms the suspected anatomy and demonstrated mild distension of the common bile duct. The gallbladder was removed and sent for histopathological examination. It was $6.7 \mathrm{~cm}$ x 3.0 $\mathrm{cm} \times 2.2 \mathrm{~cm}$ in size with associated cholelithiasis and chronic inflammatory changes.

Postoperatively the patient remained stable throughout her course and received daily packing changes, ertapenem, and eventual negative pressure wound therapy placement. The patient was discharged on day 5 post-operation with subsided drainage, early granulation tissue, and improving wound erythema as seen in Figure 5. The patient was sent home with negative pressure wound therapy, education material, and an emphasis on the physician follow-up. 


\section{Cureus}

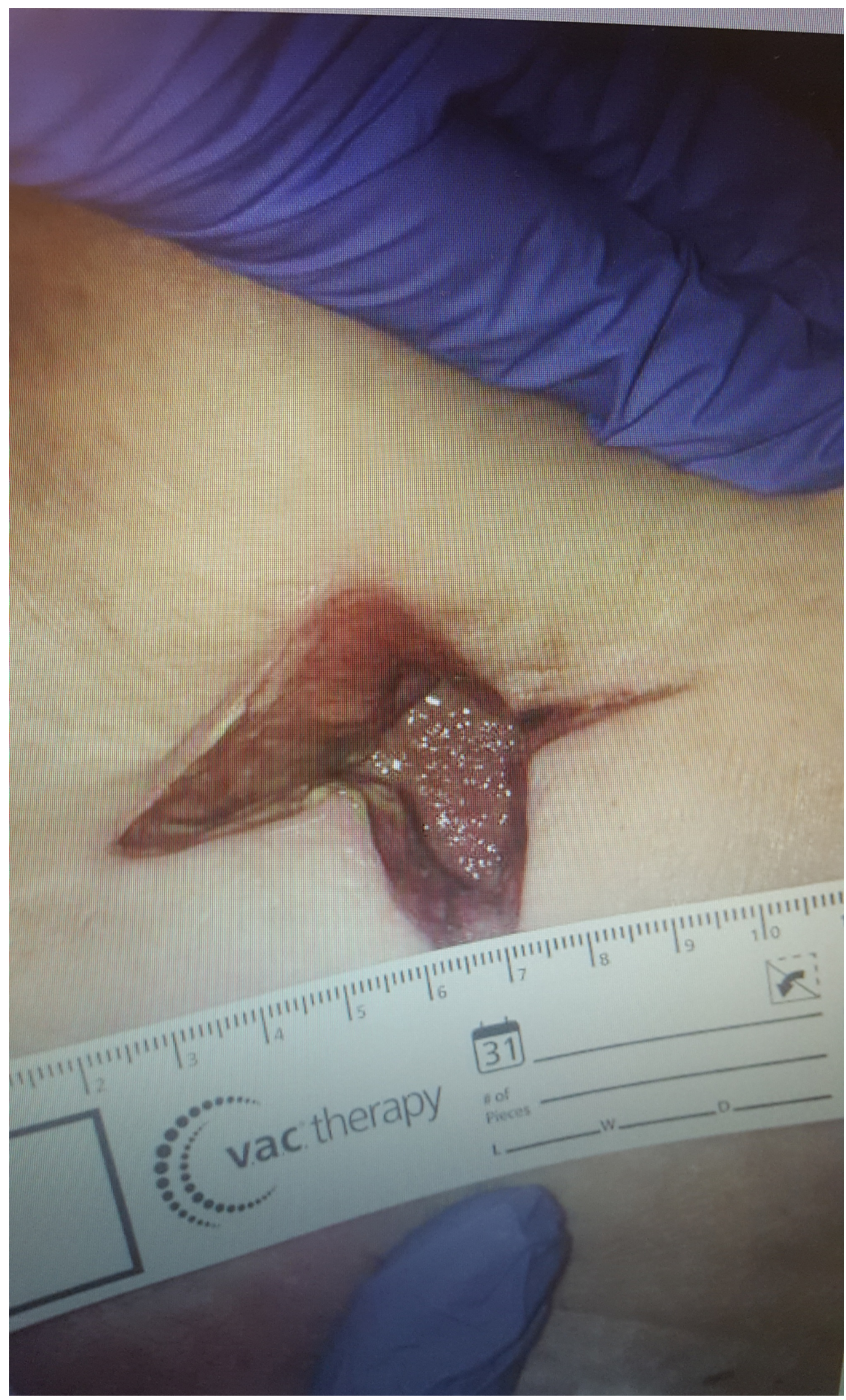

FIGURE 5: Incision site appearance day 7 after cholecystectomy with fistulotomy. Note early granulation 


\section{Discussion}

Biliary fistulas can be divided into the following categories: primary or secondary. Primary fistulas are generally associated with chronic cholelithiasis or biliary cancer, whereas secondary fistulas are associated with abdominal procedures. Biliary fistulas can further be broken down into external or internal categories, with the majority occurring internally. Internal connections include the duodenum, colon, stomach, jejunum, and common bile duct [23,6,10,12-15]. The CCF seen in this case, is a type of an EBF, which appears secondary to percutaneous drain placement with associated chronic cholecystitis.

Upon literature review, only one other case of a CCF associated with percutaneous cholecystostomy drain placement was noted [12]. In that study, the patient's fistula was discovered due to visible purulent drainage and multiple gallstones protruding out of the previous percutaneous drain site. Subsequently, the patient had their drain removed during a separate hospitalization prior to elective cholecystectomy, allowing for continued inflammation and fistula formation, similar to our case. The patient in that study was successfully treated with a laparoscopic cholecystectomy as well. One major point of contrast between the two cases is how the fistula presented itself externally. Our case presented as an abdominal wall abscess rather than a draining sinus with extraversion of stones. Although there are other reports of CCFs presenting in as abdominal wall abscesses in the literature, this is the first one noted after percutaneous cholecystostomy tube placement and removal $[4,7-8,15]$.

Percutaneous cholecystostomy drains are used in patients whose condition prevent them from having a cholecystectomy. They have been shown to be a safe and effective temporary treatment for patients with acute cholecystitis with severe comorbid conditions. Generally, the placement of these drains reduce inflammation and manage comorbidities prior to definitive treatment by cholecystectomy [16-17]. Most patients are scheduled for and follow up with their surgeons for a cholecystectomy during which they remove the drain. This unfortunately wasn't seen in this patient as she had the perfect nidus for a fistula, a preformed tract with a continually inflamed gallbladder. Known complications of percutaneous cholecystostomy placement include drain dislodgement, biliary peritonitis, pneumothorax, and colonic perforation [16-17].

CCFs are generally treated with cholecystectomy, but some are left to close on their own depending on the patient's comorbidities [18-19] In this case, due to the chronic nature of her gallbladder inflammation and recurrence of drainage from her abscess, she was treated via robotic-assisted cholecystectomy. This minimally invasive approach to cholecystectomy with CCF removal is associated with fewer risks to the patient compared with an open procedure and was preferred in this case due to the patient's extensive morbid obesity and comorbidities.

\section{Conclusions}

Although CCFs were historically a common outcome of gallbladder disease, they are now rarely seen due to improvements in the identification and treatment of gallbladder pathology. In this report, we presented a 60 -year-old morbidly obese female who developed a CCFs secondary to continued cholecystitis with an associated preformed tract from prior cholecystostomy drain placement. The authors of this study hope that this case presentation will educate other clinicians to explore the diagnosis of an EBF when presented with a RUQ abdominal abscess.

\section{Additional Information}




\section{Disclosures}

Human subjects: Consent was obtained by all participants in this study. West Virginia University issued approval 1903502177. Title: Abdominal Wall Abscess Secondary to Cholecystocutaneous Fistula via Percutaneous Cholecystostomy Tract IRB protocol number: 1903502177 he West Virginia University Institutional Review Board approved the abovereferenced protocol on 27-Mar-2019. To access this protocol, click on the protocol number link provided. Your approval correspondence concerning this action can be found in the correspondence section HERE. For more information, see the Overview Guide. Any future protocol action requests can be completed through the WVU+kc system. . Conflicts of interest: In compliance with the ICMJE uniform disclosure form, all authors declare the following: Payment/services info: All authors have declared that no financial support was received from any organization for the submitted work. Financial relationships: All authors have declared that they have no financial relationships at present or within the previous three years with any organizations that might have an interest in the submitted work. Other relationships: All authors have declared that there are no other relationships or activities that could appear to have influenced the submitted work.

\section{Acknowledgements}

The authors would like to acknowledge the patient on whom the case report was written for consent to publish. The authors would also like to thank the following peer editors for their contributions: Dr. Jocelyn Mann DO, Dr. Suzanne Courtney DO, Dr. Brittanie West DO, Dr. David Wallace DO, Dr. Warren Chin DO, Dr. Ashley N. Rubin DO, and Dr. Daniel C. Sprando MD.

\section{References}

1. Behrend A, Cullen ML: Cholesysto-choledochal fistula: an unusual form of internal biliary fistula. Ann Surg. 1950, 132:297-303.

2. Crespi M, Montecamozzo G, Foschi D: Diagnosis and treatment of biliary fistulas in the laparoscopic era. Gastroenterol Res Pract. 2016, 1-6. 10.1155/2016/6293538

3. Judd ES, Burden VG: Internal biliary fistula. Ann Surg. 1925, 81:305-312.

4. Birch BR, Cox SJ: Spontaneous external biliary fistula uncomplicated by gallstones . Postgrad Med J. 1991, 67:391-392. 10.1136/pgmj.67.786.391

5. Poudel R, Sibakoti YC, Basnet RB: Spontaneous cholecystocutaneous fistula: a case report . PMJN. 2012, 12:51-54.

6. Sulakshane S, Thakare V, Dumbre R: Consequences of gallbladder inflammation: spontaneous cholecystocutaneous fistula: a case report. Int J Surg. 2018, 5:1974-1977. 10.18203/23492902.isj20181623

7. Rinzivillo NMA, Danna R, Leanza V, et al.: Case report: spontaneous cholecystocutaneous fistula, a rare cholethiasis complication. 2017, 6:1-8. 10.12688/f1000research.12235.1

8. El Tinay O, Siddiqui ZR, Alhedaithy M, Kharashgah MNM: Right hypochondrial abscess: a rare consequence of supportive cholecystitis. Ann Med Surg (Lond). 2016, 12:106-108.

10.1016/j.amsu.2016.11.009

9. Flora HS, Bhattacharya S: Spontaneous cholecystocutaneous fistula. HPB (Oxford). 2001, 3:279-280. 10.1080/136518201753335584

10. Abbey R: Spontaneous cholecystocutaneous fistula. Int J Adv Integ Med Sci. 2016, 1:196-198.

11. Nicholson T, Born MW, Garber E: Spontaneous cholecystocutaneous fistula presenting in the gluteal region. J Clin Gastroenterol. 1999, 28:276-277.

12. Pripotnev S, Petrakos A: Cholecystocutaneous fistula after percutaneous gallbladder drainage . Case Rep Gastroenterol. 2014, 8:119-122. 10.1159/000362360

13. Morissey KP, McSherry CK, Czerniak A, Blumgart LH: Internal biliary fistula and gallstone ileus, surgery of the liver and biliary tract. Surgery of the Liver and Biliary Tract. Churchill Livingstone, Edinburgh; 1994. 1:858-860.

14. Natesan K: A rare complication of a common disease - anterior abdominal wall abscess due to spontaneous rupture of gall bladder. University Journal of Surgery and Surgical Specialities. 2017, 3:99-102. Accessed: 6/18/2018: http://ejournal- 


\section{Cureus}

tnmgrmu.ac.in/index.php/surgery/article/view/4108.

15. Ongso Y, Beh HN: Cholecystocutaneous abscess: a rare presentation of a common problem . Clin Med Insights Gastroenterol. 2017, 8:44-45. 10.4081/gi.2017.6899

16. Chopra S, Dodd III GD, Mumbower AL, et al.: Treatment of acute cholecystitis in noncritically ill patients at high surgical risk. AJR Am J Roentgenol. 2001, 176:1025-1031. 10.2214/ajr.176.4.1761025

17. Davis CA, Landercasper J, Gundersen LH, Lambert PJ: Effective use of percutaneous cholecystostomy in high-risk surgical patients techniques, tube management, and results. Arch Surg. 1999, 134:727-732. 10.1001/archsurg.134.7.727

18. Malik AH, Nadeem M, Ockrim J: Complete laparoscopic management of cholecystocutaneous fistula. Ulster Med J. 2007, 76:166-167.

19. Almotlaq AM: Laparoscopic management of a cholecystocutaneous fistula: a case report . J Minim Invasive Surg Sci. 2015, 4:1-3. 